\title{
On Optimal Replacement Thresholds with Technological Expectations ${ }^{1}$
}

\author{
Laura J. Kornish \\ The Fuqua School of Business, Duke University, \\ Box 90120, Durham, North Carolina 27708-0120 \\ kornish@mail.duke.edu \\ Received September 24, 1998; revised July 12, 1999

\begin{abstract}
Y. Balcer and S. A. Lippman (1984, J. Econ. Theory 34, 292-318) develop a model to analyze the "buy or wait" problem under technological change. They show that this dynamic problem has a threshold solution: if the difference between the best available technology and that currently held exceeds a certain threshold, then buy. They also claim that the threshold is increasing in the "discovery potential." That is, the faster the technology is changing, the higher the threshold. In this note, we point out an error in the proof of that theorem and further, we provide a numerical counterexample to the claim. Journal of Economic Literature Classification Numbers: O32, O33. (C) 1999 Academic Press
\end{abstract}

\section{INTRODUCTION}

Balcer and Lippman [1] develop a model to analyze the decision of whether to buy a current technology or to wait for a new one. In focusing on the role of technological expectations, they allow for multiple sequential innovations and multiple sequential purchases. In the model, the technological process evolves by moving among "discovery potentials." Each discovery potential can have a different distribution for size of and time until the next discovery.

Their paper presents three theorems on properties of the optimal solution to the dynamic decision problem. Theorem 1 states that the optimal solution has a threshold form. If the difference between the best available technology and that currently held exceeds a certain threshold, then the

\footnotetext{
${ }^{1}$ This research was supported in part by the Lieberman Fellowship from Stanford University. The author is grateful for helpful comments from an anonymous referee.
} 
optimal solution is to buy; otherwise, the optimal solution is to wait. Theorem 3 says that if the discovery potential is higher (i.e., technology is improving more rapidly), then the threshold is higher. In this note, we point out an error in the proof of Theorem 3 and further, we provide a counterexample to the result.

\section{MODEL}

Much of the description of the mathematical model we will borrow directly from the original paper, as it is explained well and concisely there.

[W] characterize the probabilistic evolution of the technological process in terms of three factors: (a) the current state of technology or technological knowledge,... $y$; (b) the discovery potential, a measure of the rapidity of future innovations or the pace of technological progress, labeled $i$; and (c) the time $n$ since the last discovery or technological innovation. [1, p. 296]

[T] he Markovian system evolves as follows. First, starting from a given state of knowledge $y$ and discovery potential $i$, a random amount $T_{i}$ of time elapses during which the state of knowledge and the discovery potential remain unchanged. At time $T_{i}$, the discovery potential changes (with probability $P_{i j}$ ) to level $j$ and simultaneously the state of knowledge becomes $y+Z_{i}$ (the exact value of $Z_{i}$ is observed immediately). [1, p. 298]

The random variable $Z_{i}$ has cumulative distribution function $F_{i}$. They define $V(i, z, n)$ as

the present value of the net benefits associated with improvements in the firm's technology when the discovery potential is $i$, the firm's technology lags technological knowledge by $z(=y-x)$, an amount of time $n$ has elapsed since the last scientific discovery, and the firm acts optimally with respect to its goal of maximizing its expected discounted profits. [1, p. 300]

Because the error presented below can be shown without uncertainty in discovery time, the rest of the presentation will be done using the assumption that a discovery happens every period. With this assumption, we drop the third argument of the value function $V$. Other than that simplification, we follow the notation of Balcer and Lippman (or B\&L for brevity), 


$$
\begin{aligned}
V(i, z) & =\max (-K+\Delta z ; h(i, z))+H(i) \\
h(i, z) & =\beta\left[E_{i} V(J, U+z)-E_{i} V(J, U)\right] \\
H(i) & =\beta E_{i} V(J, U) \\
E_{i} V(J, U+z) & =\sum_{j} P_{i j} \int V(j, u+z) d F_{i}(u),
\end{aligned}
$$

where $K$ is the (constant) price of a technology purchase. The one-period discount factor is $\beta$. A switching cost of $s$ is allowed, where $s$ is proportional to the size of the technological lag, and $\Delta$ is $(1 /(1-\beta))-s$. Equation (1) for $V(i, z)$ gives the value function when the discovery potential is $i$ and the lag is $z$. Equation (2) for $h(i, z)$ gives the value of the optimal future strategy if you do not buy now net of the value of the optimal future strategy if you do buy now. Equation (3) for $H(i)$ gives the value of the optimal future strategy if you do buy now. Equation (4) for $E_{i} V(J, U)$ gives the expected optimal value over the possible sizes of the discovery $u$ and the next possible state $j$.

In anticipation of Theorem 3, further definition is given to the meaning of a higher discovery potential. Saying that discovery potential $i+1$ is "higher" than discovery potential $i$ means that

1. The discovery size for $i+1$ is stochastically larger: $F_{i}(z) \geqslant F_{i+1}(z)$ for all $z$ [1, Eq. (9)].

2. "[T]he discovery potential at the time of the next innovation increases (stochastically) with the current level of discovery potential" [ 1 , p. 305]. $\sum_{j=k}^{\infty} P_{i j}$ is nondecreasing in $i$ for each fixed $k$ [1, Eq. (10)].

3. The hazard rate of time until a new discovery is not lower in every period for level $i+1$. (The multiple mathematical statements of this concept [1, Eq. $(11 \mathrm{a}, \mathrm{b}, \mathrm{c})$ and (12)] are all met by our assumption of a discovery every period.)

At this point, all of the terms and relationships in the model are defined and we can proceed to the analysis of the theorem.

\section{ERRORS REVEALED}

Balcer and Lippman show in Theorem 1 that the optimal solution has a threshold form. For each discovery potential $i$, there is a threshold $\xi_{i}$ above which it is optimal to buy. In this section, we look at the relationship between the threshold and the discovery potential. We use the notation and equation numbers from the original paper. 
THEOREM 3 (Balcer and Lippman [1]). If the structure of the technological process satisfies (9), (10), and (11c) and $T_{i}$ is not a function of $i$, then $\xi_{i} \leqslant \xi_{i+1}$ for all $i$; that is, the critical numbers $\xi_{i}$ increase with the pace of technological innovation.

The proof provided for Theorem 3 in the original paper uses an induction approach. The proof is by induction on $m$, the number of decision periods left. They define (for the case with a discovery every period)

$$
V_{m+1}(i, z)=\max \left(-K+\Delta z ; h_{m}(i, z)\right)+H_{m}(i),
$$

where $V_{0}(i, z)=0$ and $h_{m}$ and $H_{m}$ are defined as above, except that $V_{m}$ replaces $V$.

The proof provided tries to show that $\partial V_{m}(i, z) / \partial z$ and $\partial h_{m}(i, z) / \partial z$ are nondecreasing in $i$, the discovery potential. The induction proceeds as follows. Assume that $\partial V_{m}(i, z) / \partial z$ a nondecreasing function of $i$. They demonstrate that it follows that $\partial h_{m}(i, z) / \partial z$ is a nondecreasing function of $i$. Then they claim that the recursion for $V_{m+1}$ above implies that $\partial V_{m+1}(i, z) / \partial z$ is a nondecreasing function of $i$.

The last statement contains the fundamental error. Given that $a(k, x)=\max (b(x), c(k, x))$, knowing that $\partial c(k, x) / \partial x$ is nondecreasing in $k$ does not imply that $\partial a(k, x) / \partial x$ is nondecreasing in $k$ for all $x$. See Fig. 1. This fundamental error hides other errors: $\partial V_{m} / \partial z$ and $\partial h_{m} / \partial z$ are actually

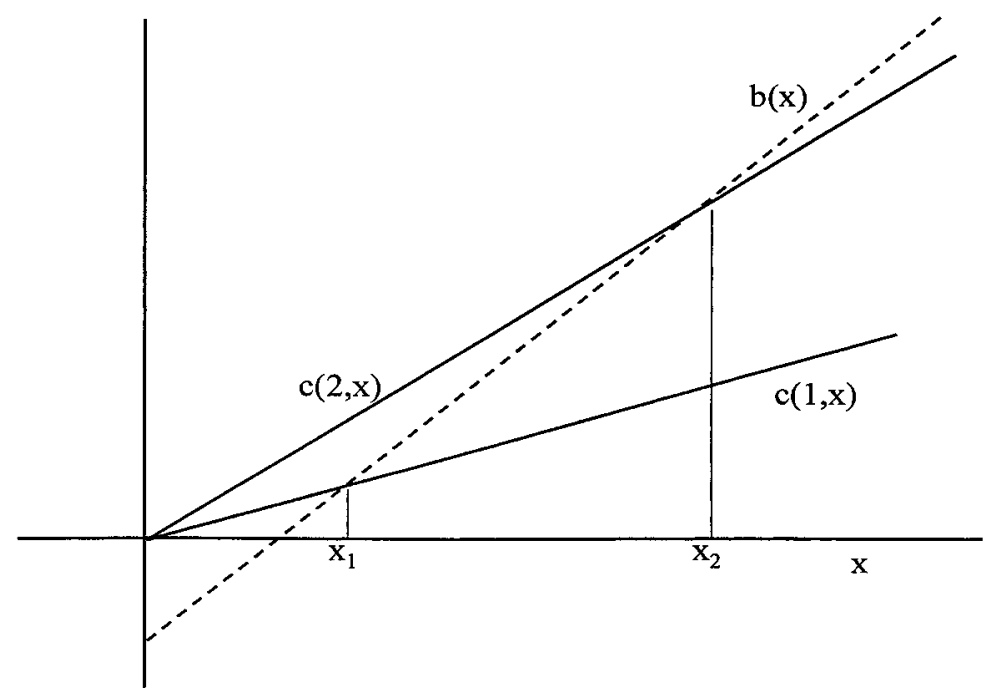

FIG. 1. If $a(k, x)=\max (b(x), c(k, x))$, then between $x_{1}$ and $x_{2}, a(1, x)$ has a higher slope than $a(2, x)$. 
not increasing in $i$ for all $z$. There are lags for which the marginal value of extra lag is higher in a lower discovery potential.

\section{NUMERICAL COUNTEREXAMPLE}

We present a simple numerical example to show that the threshold is not necessarily increasing in the discovery potential. The example has only integer-sized discoveries; this structure allows us to solve a Markov decision problem with a policy improvement algorithm [2]. The state is the combination of discovery potential and lag between the best available technology and that currently held.

The example has three discovery potential levels. The discovery size distributions are degenerate. In discovery potential 1 , there is a discovery of size 1 each period; in discovery potential 2, there is a discovery of size 2 each period; in discovery potential 3 , there is a discovery of size 2 each period. The cost of purchase $K$ is 10 . The one-period discount factor $\beta$ is 0.9 . The switching cost $s$ is 0 . The one-step transition matrix for transitions between discovery potentials is

$$
P_{i j}=\left(\begin{array}{lll}
0.7 & 0.2 & 0.1 \\
0.5 & 0.3 & 0.2 \\
0.4 & 0.3 & 0.3
\end{array}\right) .
$$

The state space is limited by the fact that for lags at or above the purchase price, the optimal decision is to buy. The optimal values are shown in Table I.

One can see that although $V(i, z)$ is increasing in both $i$ and $z$, the differences $V\left(i, z^{\prime}\right)-V(i, z), z^{\prime}>z$, are not all increasing in $i$. In particular, $V(2,3)-V(2,2)=8.48>V(3,3)-V(3,2)=8.40$. This lack of monotonicity in discovery potential of the difference $V\left(i, z^{\prime}\right)-V(i, z)$ makes Eqs. (9) and

\section{TABLE I}

Optimal Values for the (Discovery Potential, Lag) Combinations

\begin{tabular}{rrrrrrrrrrr}
\hline & \multicolumn{10}{c}{$z$} \\
\cline { 2 - 10 } & \multicolumn{1}{c}{1} & \multicolumn{1}{c}{2} & 3 & 4 & 5 & 6 & 7 & 8 & 9 \\
\hline$V(1, z)$ & 91.52 & 98.68 & 106.42 & 115.06 & 124.92 & 134.92 & 144.92 & 154.92 & 164.92 \\
$V(2, z)$ & 100.64 & 108.36 & 116.84 & 125.41 & 134.39 & 143.39 & 153.35 & 163.35 & 173.35 \\
$V(3, z)$ & 101.66 & 109.37 & 117.77 & 126.25 & 135.24 & 144.31 & 154.31 & 164.31 & 174.31 \\
\hline
\end{tabular}


TABLE II

Optimal Thresholds for Counterexample to

Balcer and Lippman Theorem 3

\begin{tabular}{cc}
\hline Discovery potential & Threshold \\
\hline 1 & 5 \\
2 & 7 \\
3 & 6 \\
\hline
\end{tabular}

(10) of $\mathrm{B} \& \mathrm{~L}$ insufficient to show that $h(i, z) \equiv \beta \sum_{j} P_{i j} \int[V(j, u+z)-$ $V(j, u)] d F_{i}(u)$ is a nondecreasing function in $i$.

The optimal strategy can be deduced by comparing the optimal values to the values for the buy option, $-K+\Delta z+H(i)$. The optimal strategies are shown in Table II, and they are not increasing.

\section{DISCUSSION}

Balcer and Lippman intuitively argue that Theorem 3 makes sense because things are changing faster, so the consumer wants to avoid getting locked in. In fact, it is not true in general that the thresholds are higher when the process is in a higher discovery potential. It is true that optimal values are higher for higher discovery potentials. However, the base value of what you will have next time whether you buy or not is higher, too. The threshold is determined by the difference of these two quantities, optimal value minus base value. Both are increasing in discovery potential, but the difference does not necessarily have that property.

Balcer and Lippman's model focuses on the importance of technological expectations in an equipment replacement problem under technological change. The revelation of the error in one of the theorems challenges us to further characterize the optimal solution to the "buy or wait" problem when indefinitely many technologies are forecast.

\section{REFERENCES}

1. Y. Balcer and S. A. Lippman, Technological expectations and adoption of improved technology, J. Econ. Theory 34 (1984), 292-318.

2. R. A. Howard, "Dynamic Programming and Markov Processes," Technology Press, Cambridge, MA, 1960. 